\title{
Leptospirosis porcina: prevalencia serológica en establecimientos productores de la República Argentina
}

\author{
Swine leptospirosis: Serological prevalence inproducing \\ establishments in Argentina Republic
}

\author{
Jessica Petrakovsky M, ${ }^{1 *}$ Lic, Julio Tinao, ${ }^{1}$ Tec, Jorge Esteves $\mathrm{M},{ }^{1} \mathrm{MV}$. \\ ${ }^{1}$ Laboratorio de Referencia de la O.I.E. en Leptospirosis. Dirección de Laboratorios y Control Técnico, \\ SENASA. Buenos Aires. Argentina. Correspondencia:jpetrako@senasa.gov.ar
}

Recibido: Enero de 2012; Aceptado: Septiembre de 2012.

\section{RESUMEN}

Objetivo. Detectar la presencia de anticuerpos contra Leptospira sp en cerdos que no presentaban sintomatología clínica, pertenecientes a establecimientos de producción porcina ubicados en todo el territorio de la República Argentina. Materiales y métodos. La asignación de predios y muestras se realizó en forma proporcional a los registrados en cada provincia a lo largo de todo el país. En total se estudiaron 1.524 predios, de los cuales se tomaron 19.872 muestras de suero y en la primera etapa se procesaron 3.631 de los mismos. Este estudio toma en consideración zonas identificadas por provincia, municipio y predios. Los sueros fueron analizados por la técnica de MAT (prueba de microaglutinación). Resultados. El 30\% de las muestras resultaron positivas a la prueba tamiz empleada (primera etapa del MAT). La mayoría presentó coaglutininas a dos o a tres serovariedades. Al realizar la titulación de los sueros (segunda etapa del MAT), el porcentaje de positividad se mantuvo, siendo los serovares de mayor prevalencia Castellonis e Icterohaemorrhagiae. El 90\% de las muestras presentó un título final de 1:200. Conclusiones. La existencia de sueros positivos en todas las provincias indica el contacto de los animales con la leptospira a lo largo de todo el país.

Palabras clave: Cerdo, epidemiología, leptospira, leptospirosis (Fuente: Agrovoc).

\section{ABSTRACT}

Objective. To detect the presence of antibodies to leptospira $s p$ in pigs that didn't show clinical symptoms, of swine production farms located throughout Argentina. Materials and methods. The location of farms and samples were chosen in proportion to those recorded in each province covering the whole country. The number of farms studied was 1,524 , with 19,872 serum samples. This study takes into consideration areas identified by provinces, municipalities and properties. In the first phase 3,631 sera were processed and analyzed by MAT (microscopic agglutination test). Results. The $30 \%$ of the samples tested were positive for the screening test (MAT). Most of these samples presented coagglutinins for two or three serovars. In the titration of sera, the percentage of positives was the same, the most prevalent serovars were Icterohaemorrhagiae and Castellonis. The $90 \%$ of the samples had a final titer of 1:200. Conclusions. The existence of positive sera in all provinces indicates the contact of animals with leptospira throughout the country.

Key words: Epidemiology, leptospira, leptospirosis, pigs (Source: Agrovoc). 


\section{INTRODUCCION}

La leptospirosis es una zoonosis infectocontagiosa de nivel mundial producida por cepas patógenas del género Leptospira (1). La enfermedad es más frecuente en regiones de clima tropical o subtropical debido a las altas condiciones de humedad que son necesarias para la supervivencia de las leptospiras. La epidemiología de la leptospirosis ha sido modificada por los cambios en la cría de animales, el clima y el comportamiento humano (2).

La enfermedad se mantiene en la naturaleza debido a la infección crónica de los túbulos renales de animales portadores, que excretan el microorganismo en la orina, contaminando el medio ambiente. La infección humana se produce por contacto directo con la orina o los tejidos de animales infectados o, más comúnmente por la exposición indirecta a las leptospiras en el suelo húmedo o agua. Los reservorios más importantes son los roedores y otros pequeños mamíferos, pero los animales de compañia y el ganado, también son fuentes significativas para el contagio de los humanos. La infección de los animales portadores por lo general se produce durante edades tempranas y, una vez infectados, pueden excretar leptospiras en la orina en forma intermitente o continua durante toda la vida (3).

Los porcinos criados en sistemas intensivos plantean un problema diferente a los criados a campo o semi intensivos. En los grandes criaderos la posibilidad de infección cruzada es muy importante debido a la alta densidad de población. El movimiento de los animales de un corral a otro y el contacto con desechos de otros corrales son los medios más importantes de diseminación de la enfermedad en estos establecimientos. La introducción de la misma puede ocurrir por la incorporación al plantel de un padrillo que sea portador de leptospira en su aparato genital. Los animales susceptibles adquieren la infección por contacto directo o indirecto (4).

El conocimiento de los serotipos prevalentes y de los hospedadores de mantenimiento, es esencial para entender la epidemiología de la enfermedad en cualquier región (5).

En Argentina la leptospirosis tiene un impacto económico negativo en las producciones porcinas y bovinas, con formas agudas o crónicas, acompañadas de abortos y muertes perinatales, retención placentaria, infertilidad, anemia hemolítica, ictericia y congestión pulmonar, así como disminución de la producción láctea en bovinos de leche. (6)
Considerando que los servicios veterinarios oficiales tienen un importante rol en el control de la leptospirosis, los laboratorios de referencia les brindan apoyo técnico en el diagnóstico y en la aplicación de distintas medidas de control. A partir de un importante muestreo llevado a cabo por el SENASA, este Laboratorio ha iniciado un estudio de prevalencia en la especie porcina. Los sueros a los que se tiene acceso se encuentran perfectamente identificados por provincia, municipio y predio.

El objetivo del presente trabajo fue detectar la presencia de anticuerpos contra leptospira en cerdos que no presentaban sintomatología clínica, distribuidos en distintos puntos de la República de Argentina.

\section{MATERIALES Y MÉTODOS}

Tipo de estudio. Se realizó un estudio descriptivo de corte transversal. La población bajo estudio comprendió una muestra representativa de la totalidad de la población porcina doméstica local. Los sueros analizados provinieron en su origen de un muestreo nacional realizado para determinar la prevalencia de peste porcina.

Sitio de estudio. Este muestreo se realizó en el año 2006 y cubrió la totalidad del País. Se tuvo en cuenta la cantidad de predios por provincia y el número de animales contenidos en cada uno de ellos. La asignación de predios o muestras se realizó en forma proporcional a los registrados en cada provincia.

Muestra. El total de predios muestreados fue 1.524, donde se obtuvieron 19.872 muestras de suero de los cuales se analizaron 3631 . Cada muestra fue georreferenciada teniendo en cuenta provincia, partido y predio.

Población. Se define la población como el universo de cerdos existentes en el momento del muestreo antes aludido. Al conglomerado de área como las provincias, partidos y predios con existencia de especie porcina. A la variable en estudio como la proporción de cerdos con presencia o ausencia de anticuerpos contra leptospiras, y a la unidad de muestreo como el suero individual de cada cerdo.

Determinación del tamaño de la muestra. En función a lo expuesto, y tomando en cuenta que los antecedentes de prevalencia de leptospirosis porcina en Argentina son muy variables (7), se calculó el tamaño de la muestra para detectar 
una prevalencia del $50 \%$ con un error del $2.5 \%$ y una confianza del $95 \%$. El cálculo se realizó mediante el programa Win Episcope 2.0 (8), el cual determinó que se deben tomar por lo menos 1.427 muestras. Dado que nuestra capacidad operativa permitía aumentar ese número se procesaron, en esta primera etapa, 3.631 muestras a fin de disminuir los errores muestrales (dado las características de la población en estudio).

Análisis de los sueros. Con las muestras obtenidas se realizó una búsqueda de anticuerpos contra leptospirosis utilizando la prueba de microaglutinación (MAT) con el uso de una batería de cinco serovariedades de Leptospira interrogans de referencia (Castellonis, Icterohaemorrhagiae, Pomona, Pyrogenes y Wolffi) que en estudios anteriores mostraron estar presentes en Argentina (7). Estas cepas fueron cultivadas en medio líquido EMJH (Difco, Ref 279410) (9).

El procedimiento utilizado en la prueba de microaglutinación (MAT) fue el descrito en el "Manual de las Pruebas de Diagnóstico y de las Vacunas para los Animales Terrestres. OIE. 2008". El criterio de positividad fue un título mayor o igual a 1:100. En una primera etapa se procesaron los sueros diluidos 1:100 para identificar los positivos, los cuales fueron titulados posteriormente para determinar el serovar presente en ese suero y el valor de su título. Cuando se utiliza la dilución del suero 1:100 pueden detectarse como positivos más de un serovar, debido a la presencia de coaglutininas (10).

\section{RESULTADOS}

De los 3.631 animales muestreados, 1.102 resultaron positivos, es decir el $30 \%$ de las muestras resultaron positivas a la dilución 1:100 (Tabla 1a). En esta etapa, la mayoría de los sueros reaccionaron a dos (46\%), o a tres o más serovares (45\%) (Tabla 1 b). Los serovares que aparecieron con más frecuencia fueron Castellonis (65\%), Wolffi (49.1\%) y Pomona e Icterohaemorrhagiae (45\%) (Tabla 1c). Los porcentajes obtenidos suman más de 100 , debido a que algunos sueros han dado positivos a más de un serovar.

En la titulación final se observó que se mantuvo el porcentaje de positividad (30\%), siendo los serovares de mayor prevalencia Castellonis con un $33.7 \%$ e Icterohaemorrhagiae con el $23.6 \%$ (Tabla 2).
Tabla 1a. Cantidad de animales positivos y negativos y sus respectivos porcentajes.

\begin{tabular}{cccc}
\hline & \multicolumn{3}{c}{ Animales muestreados } \\
\cline { 2 - 4 } & Positivos & Negativos & Total \\
\hline NM & 1.102 & 2.509 & 3611 \\
P & 30.35 & 69.65 & 100.0 \\
\hline
\end{tabular}

Tabla 1b. Cantidad de serovares hallados por animal y sus respectivos porcentajes.

\begin{tabular}{cccc}
\hline & $\mathbf{1}$ & $\mathbf{2}$ & $\begin{array}{c}\text { 3 o } \\
\text { más }\end{array}$ \\
\hline $\mathrm{NM}$ & 94 & 512 & 496 \\
$\mathrm{P}$ & 8.53 & 46.46 & 45.01 \\
\hline
\end{tabular}

Tabla 1c. Frecuencia de aparición de cada serovar analizado, expresada en números absolutos y porcentajes. Los porcentajes suman más de 100 debido a que cada serovar puede aparecer más de una vez en screenig.

\begin{tabular}{cccccc}
\hline & \multicolumn{5}{c}{ Serovar de L. Interrogans } \\
\cline { 2 - 6 } & Castellonis & $\begin{array}{c}\text { Icterohae- } \\
\text { morrhagiae }\end{array}$ & Pomona & $\begin{array}{c}\text { Pyroge- } \\
\text { nes }\end{array}$ & Wolffi \\
\hline NM & 766 & 494 & 496 & 310 & 541 \\
$\mathrm{P}$ & 69.5 & 44.8 & 45.0 & 28.1 & 49.1 \\
\hline
\end{tabular}

NM: NUM. MUESTRAS; P: PORCENTAJES

Los $\%$ suman mas de $100 \% \times q$ se refieren al total de + . Algunos sueros se manifiestan + a mas de un serovar.

Tabla 2. Resultados finales obtenidos en la titulación.

\begin{tabular}{ccccccc}
\hline & \multicolumn{5}{c}{ Positivos por titulación Serovar de $L$. Interrogans } \\
\cline { 2 - 7 } & $\begin{array}{c}\text { Caste- } \\
\text { Ilonis }\end{array}$ & $\begin{array}{c}\text { Icterohae- } \\
\text { morrhagiae }\end{array}$ & $\begin{array}{c}\text { Pomo- } \\
\text { na }\end{array}$ & $\begin{array}{c}\text { Pyro- } \\
\text { genes }\end{array}$ & Wolffi & Total \\
\hline NM & 371 & 260 & 186 & 110 & 175 & 1.102 \\
P & $33.7 \%$ & $23.6 \%$ & $16.9 \%$ & $10.0 \%$ & $15.8 \%$ & $100.0 \%$ \\
\hline
\end{tabular}

Los títulos obtenidos fueron bajos, casi la totalidad de las muestras (90\%), presentó títulos de 1:200 (Tabla 3, y Figura 1).

Tabla 3. Frecuencia de aparición de los títulos finales positivos (iguales o mayores a $1: 100$ ) expresados en números absolutos y porcentajes.

\begin{tabular}{cccccc}
\hline Dilución de suero & $\mathbf{1 : 1 0 0}$ & $\mathbf{1 : 2 0 0}$ & $\mathbf{1 : 4 0 0}$ & $\mathbf{1 : 8 0 0}$ & Total \\
\hline Cantidad & 77 & 993 & 31 & 1 & 1.102 \\
Porcentaje & $7.0 \%$ & $90.1 \%$ & $2.8 \%$ & $0.1 \%$ & $100.0 \%$ \\
\hline
\end{tabular}

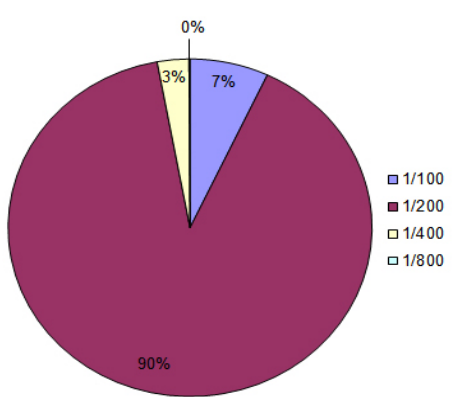

Figura 1. Porcentaje y relación de los títulos finales positivos (mayores o iguales a 1:100). 
El porcentaje de positividad de cada provincia fue variable aunque en 20 de las 22 provincias (90\%), estuvo comprendido en el rango de $20-40 \%$ (Tabla 4, Figura 2).

En la figura 3 se observan en cada provincia los serovares detectados en la primera etapa y los confirmados por titulación. El serovar prevalente fue Castellonis.

La mayoría de las muestras con serología positiva se concentraban en la región media y norte del país ya que en esas zonas es donde se ubican predominantemente las explotaciones porcinas (Figura 4).

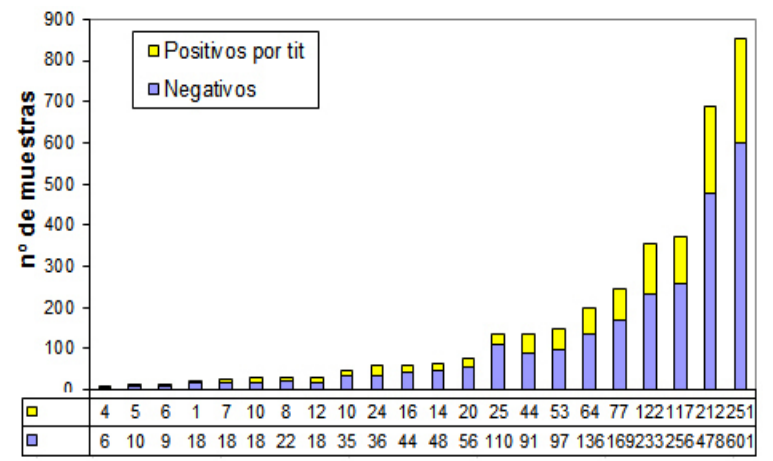

Figura 2. Frecuencia de aparición de sueros positivos y negativos expresados por provincia
Tabla 4 . Cantidad de sueros positivos (determinados en la titulación, con título mayor o igual a $1: 100$ ) y negativos en cada provincia y sus respectivos porcentajes.

\begin{tabular}{lcccc}
\hline PROVINCIA & Neg & $\begin{array}{c}\text { Positivos } \\
\text { por } \\
\text { titulación }\end{array}$ & $\begin{array}{c}\text { Porcentaje } \\
\text { de positivos }\end{array}$ & Totales \\
\hline CORRIENTES & 6 & 4 & 40 & 10 \\
MENDOZA & 10 & 5 & 33 & 15 \\
NEUQUEN & 9 & 6 & 40 & 15 \\
CATAMARCA & 18 & 1 & 5 & 19 \\
ENTRE RIOS & 18 & 7 & 28 & 25 \\
RIO NEGRO & 18 & 10 & 36 & 28 \\
SAN JUAN & 22 & 8 & 27 & 30 \\
SANTA CRUZ & 18 & 12 & 40 & 30 \\
CHACO & 35 & 10 & 22 & 45 \\
CHUBUT & 36 & 24 & 40 & 60 \\
JUJUY & 44 & 16 & 27 & 60 \\
MISIONES & 48 & 14 & 23 & 62 \\
LA RIOJA & 56 & 20 & 26 & 76 \\
SALTA & 110 & 25 & 19 & 135 \\
TUCUMAN & 91 & 44 & 33 & 135 \\
SAN LUIS & 97 & 53 & 35 & 150 \\
FORMOSA & 136 & 64 & 32 & 200 \\
LA PAMPA & 169 & 77 & 31 & 246 \\
STG. ESTERO & 233 & 122 & 34 & 355 \\
SANTA FE & 256 & 117 & 31 & 373 \\
CORDOBA & 478 & 212 & 31 & 690 \\
BUENOS AIRES & 601 & 251 & 29 & 852 \\
\hline Total general & $\mathbf{2 5 0 9}$ & $\mathbf{1 1 0 2}$ & $\mathbf{3 1}$ & $\mathbf{3 6 1 1}$ \\
\hline & & & & \\
\hline
\end{tabular}

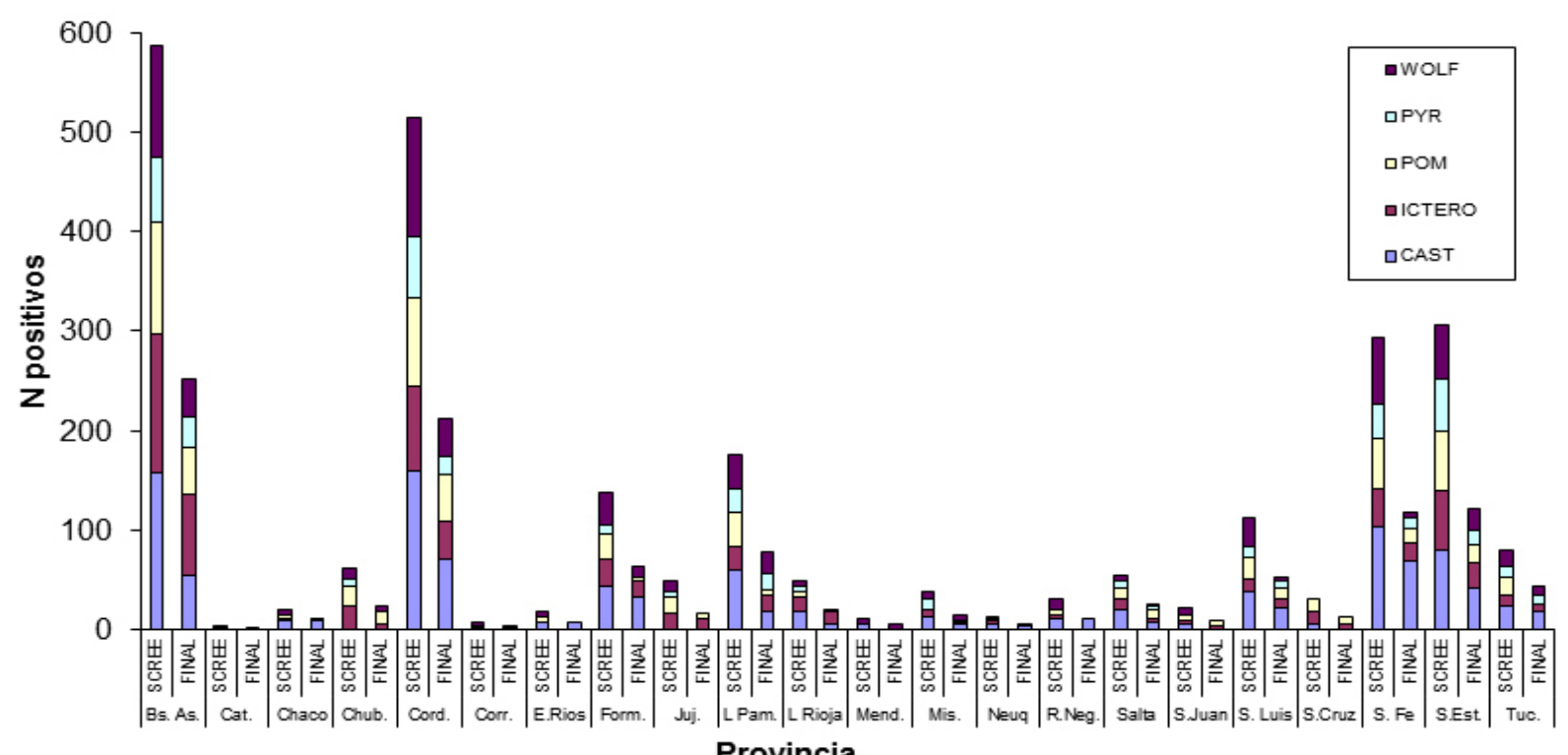

Provincia

Figura 3. Comparación entre la cantidad de sueros positivos a los distintos serovares que se detectan en el screening y la confirmada posteriormente en la titulación en cada provincia que se detectan en el screening y la confirmada posteriormente en la titulación en cada provincia. 


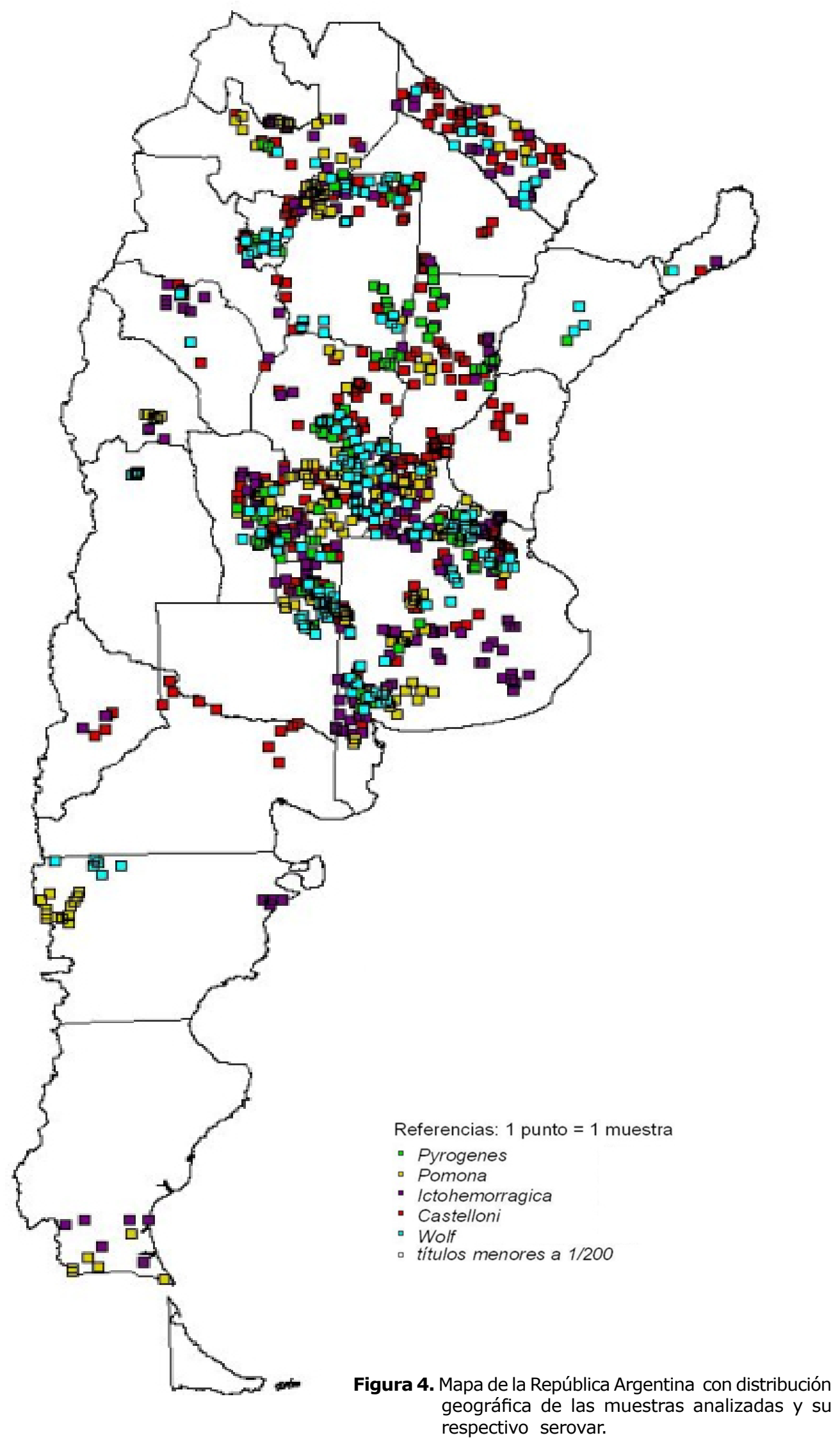




\section{DISCUSIÓN}

La existencia de anticuerpos en los sueros en todas las provincias muestreadas indica que existe un contacto de los animales con la leptospira en todo el país. A nivel nacional el $30 \%$ de los animales muestreados resultaron positivos y a nivel provincial se observó una tendencia similar.

El serovar prevalente fue Castellonis, seguido por Icterohaemorrhagiae. Existe un agrupamiento de los animales reaccionantes en la región Central y Norte del País, el cual coincide geográficamente con las áreas donde se encuentran mayor cantidad de explotaciones porcinas. También debe considerarse que en esas regiones geográficas predomina un clima cálido y húmedo propicio para el desarrollo de la Leptospira.

Es relevante conocer la prevalencia de los anticuerpos correspondientes a cada serovariedad, observar si se mantiene en el tiempo y si se agregan nuevas serovariedades a las ya existentes. Esto se basa en el comportamiento regional de la leptospirosis porcina, influenciada directamente por condiciones ambientales, por el tipo de explotación y por la fauna anexa a donde se alojan los animales $(11,12)$.

Debido a la información remitida en el presente trabajo sumada a la ya existente, sería necesario realizar otro estudio orientado a zonas que tradicionalmente no se hubieran seleccionado para la búsqueda de anticuerpos de leptospira, realizando el debido doble muestreo y orientado posteriormente a obtener aislamientos de Leptospira sp. Es fundamental contar con datos epidemiológicos en la Región para poder implementar medidas de prevención y control adecuadas, ya que la leptospirosis es una enfermedad de muy difícil erradicación debido a que se mantiene en sus reservorios silvestres.

En conclusión, la existencia de sueros positivos en todas las provincias indica el contacto de los animales con la leptospira a lo largo de todo el país.

\section{REFERENCIAS}

1. Gil A, Samartino L. Zoonosis en los sistemas de Producción Animal de las Areas Urbanas y Periurbanas de América Latina. Policy Discusión Paper No. 2. Livestock: FAO; 2002.

2. Fabré $Y$, Suárez $Y$, Rodríguez $\mathrm{O}$, Martínez $\mathrm{H}$, Feraud $D$, Cruz $M$ et al. Estudio retrospectivo de leptospirosis en la población humana y animal en municipios habaneros entre 1987-2006. Revista Salud Animal 2010; 32(3):180-187.

3. Levett PN, Haake DA. Leptospira species (leptospirosis). In: Principles and Practice of Infectious Diseases. Edited by: Mandell GL, Bennett JE, Dolin R. 7 ed. Philadelphia, Churchill Livingstone: Elsevier; 2010.

4. Adler B, De la Peña Moctezuma A. Leptospira and Leptospirosis. Vet Microbiol 2010; 140: 287-296.

5. Alonso-Andicoberry C, García-Peña FJ, OrtegaMora LM. Epidemiología, diagnóstico y control de la leptospirosis bovina. Investigación Agraria: Producción y Sanidad de los Animales. 2001; 16:205-225.

6. Levett P. Leptospirosis. Clin Microbiol Rev 2001; 14(2):296-326.

7. Llorente P, Leoni L, Martinez Vivot M. Leptospirosis en camélidos sudamericanos. Estudio de prevalencia serológica en distintas regiones de la Argentina. Arch Med Vet 2002; 34(1): 59-68.
8. Comisión Científica sobre Leptospirosis de la República Argentina. Asociación Argentina de Veterinarios de Laboratorios de Diagnóstico. Informe sobre leptospirosis en la República Argentina. Argentina: Fundación Mundo Sano; 2002.

9. Win Episcope 2.0. España: University of Zaragoza, Veterinary Faculty. Department of Infectious Diseases and Epidemiology; 2000.

10. Organización Mundial de la Salud. Anexo 16: Preparación de Medios de Cultivo. En Organización Mundial de la Salud. Leptospirosis Humana: Guía para el Diagnóstico, Vigilancia y Control. Traducción del Centro Panamericano de Fiebre Aftosa. Río de Janeiro: Centro Panamericano de Fiebre Aftosa; 2008.

11. Organización Mundial de Sanidad Animal (OIE). Leptospirosis. En: OIE, editores. Manual de las pruebas de diagnóstico y de las vacunas para los animales terrestres. Sexta edición. Paris: OIE; 2008.

12. Rosa R, Murillo N. Guía para el Control y Manejo de la Leptospirosis. Organización Panamericana de La Salud. OPS/HCP/HCV/ URU.ZOO.01/02. Uruguay: Organización Mundial De La Salud; 2002. 